\title{
Dynamics of Ku80 in Living Hamster Cells with DNA Double-Strand Breaks Induced by Chemotherapeutic Drugs
}

\author{
Manabu KOIKE ${ }^{1) *}$, Aki KOIKE ${ }^{1)}$, Jun SUGASAWA ${ }^{1)}$, Tatsushi TOYOOKA ${ }^{2)}$ and Yuko IBUKI ${ }^{2)}$ \\ ${ }^{1)}$ DNA Repair Gene Res., National Institute of Radiological Sciences, 4-9-1 Anagawa, Inage-ku, Chiba 263-8555 and \\ ${ }^{2)}$ Laboratory of Radiation Biology, Graduate School of Nutritional and Environmental Sciences, University of Shizuoka, 52-1 Yada, \\ Shizuoka 422-8526, Japan
}

(Received 28 April 2010/Accepted 5 June 2010/Published online in J-STAGE 18 June 2010)

\begin{abstract}
A variety of chemotherapeutic drugs, e.g., etoposide and bleomycin, are widely used in clinical practice to treat many types of animal malignancies. In the clinical situation, cellular resistance to chemotherapy is a significant component of tumor treatment failure. A variety of DNA repair factors, e.g., Ku80, might be a key contributor to chemoresistance to anticancer agents. In both cancer and normal cells, Ku80 plays a key role as a sensor of DNA double-strand break (DSB) induced by treatment with some chemotherapeutic drugs. Although the localization and mobility of Ku80 play a key role in regulating the physiological function of Ku80, it is not clear whether those of Ku80 are affected after treatment with chemotherapeutic drugs. We examined the localization and mobility of Ku80 in living hamster cells with or without DSBs, which were induced by treatment with chemotherapeutic drugs. Our data showed that $\mathrm{Ku} 80$, in contrast to $\mathrm{H} 2 \mathrm{AX}$, is highly mobile in the nuclei. We found that before and after the induction of DNA damage by treatment with etoposide or bleomycin, a major portion of Ku80 is exchanged by the same kinetics in the nuclei of interphase cells. These results suggest that the mobility of a major portion of Ku80 is not affected by DNA DSBs in order to find other DSBs. In addition, the information would be worthy to develop some new chemotherapeutic drugs to treat many types of animal malignancies. KEY WORDS: bleomycin, cancer, etoposide, Ku80, localization.
\end{abstract}

A DNA double-strand break (DSB) is the most dangerous DNA damage [11]. Unrepaired or improperly repaired DSBs can lead to chromosomal truncations and translocations, which can contribute to cancer in higher eukaryotic organisms. The phosphorylation of $\mathrm{H} 2 \mathrm{AX}$ occurs at large chromatin domains flanking each DSB immediately after damage induction, e.g., ionizing radiation [27, 28]. DNAdependent protein kinase (DNA-PK), as well as ATM kinase, can phosphorylate serine 139 in a variant form of histone $\mathrm{H} 2 \mathrm{~A}$ designated as $\gamma-\mathrm{H} 2 \mathrm{AX}[24,32] . \quad \gamma-\mathrm{H} 2 \mathrm{AX}$ forms foci at DNA DSBs induced by IR and drugs and plays a critical role in the retention of repair factors at sites of DNA DSBs [1, 3, 4, 28]. Two major pathways exist in mammalian cells for the repair of DNA DSBs: nonhomologous DNA-end-joining (NHEJ) repair and homologous recombination (HR) $[11,29]$. The NHEJ repair process, which is responsible for repairing a major fraction of DNA DSBs in somatic cells of all multicellular eukaryotes, is considered to begin with the binding of $\mathrm{Ku}$ [9].

$\mathrm{Ku}$ is a complex of 2 protein subunits of 70 and $80 \mathrm{kDa}$, hereafter designated as $\mathrm{Ku} 70$ and $\mathrm{Ku} 80$, respectively. It was shown that $\mathrm{Ku}$ is a DNA-binding component of a DNA-PK that phosphorylates many nuclear proteins in vitro, e.g., p53, Artemis, XRCC4, DNA-PKcs, or Ku itself [5, 21]. Accumulating evidence suggests the implication of the $\mathrm{Ku}$ in tumorigenesis and acquired drug resistance $[5,8]$. On the other hand, $\mathrm{Ku} 70, \mathrm{Ku} 80$, and DNA-PKcs knockout mice

\footnotetext{
*Correspondence to: Koike, M., DNA Repair Gene Res., National Institute of Radiological Sciences, 4-9-1 Anagawa, Inage-ku, Chiba 263-8555, Japan.

e-mail: m_koike@nirs.go.jp
}

exhibit tumorigenesis $[8,11]$. In addition, the combined loss of p53 and a genome caretaker gene, e.g., $\mathrm{Ku} 70, \mathrm{Ku} 80$, or DNA-PKcs, accelerates genomic instability and tumorigenesis [8, 11].

Defects in NHEJ result in genomic instability as evidenced by a variety of increased chromosomal aberrations [7]; it follows that defects in this pathway may potentiate malignant transformation. Severe combined immunodeficiency (SCID) is an inherited disorder of humans, mice, horses, and dogs, in which affected individuals are incapable of generating antigen-specific immune responses [6]. The defective factor in these SCID animals is DNA-PKcs. Mutations interfering with DNA-PK in C.B-17 mice, foals, and Jack Russell Terriers occur in DNA-PKcs. On the other hand, mice with DNA-PKcs deficiencies or with defects in any component of the NHEJ pathway have an increased incidence of both spontaneous and radiation induced thymic lymphoma [11]. Statistically significant correlation between the development of sarcoid tumors in Arabian horses and heterozygosity for the mutant DNA-PKcs allele has been reported [6].

DNA-PK is composed of two components: DNA-PKcs and $\mathrm{Ku}[11]$. $\mathrm{Ku}$ stimulates the recruitment to DSBs of DNA-PKcs and activates the kinase activity [11]. Thus, the localization of $\mathrm{Ku} 70$ and $\mathrm{Ku} 80$ plays a key role in regulating the physiological function of $\mathrm{Ku}$ and DNA-PKcs in vivo [12, 31]. It was reported that $\mathrm{Ku} 80$ plays a key role in the repair of DNA DSBs induced by treatment with some chemotherapeutic drugs [2]. It has not been determined whether the mobility of Ku80 is affected after treatment with these drugs, although there are many reports about the localiza- 
tion of Ku80 [12]. Previously, we generated cell lines expressing Ku80 tagged with enhanced green fluorescent protein (EGFP) using Ku80-deficient hamster cells [13]. The EGFP-Ku80 complemented not only X-ray sensitivity but also the etoposide sensitivity of Ku80-deficient cells [13].

Resistance to anticancer agents is one of the primary impediments to effective cancer therapy. Ku80 might be a key contributor to chemoresistance to anticancer agents, e.g., etoposide and bleomycin [2]. A variety of chemotherapeutic drugs, e.g., etoposide and bleomycin, are widely used in clinical practice to treat many types of animal malignancies. In the clinical situation, cellular resistance to chemotherapy is a significant component of tumor treatment failure. Ku80 plays a key role as a sensor of DNA doublestrand break (DSB) induced by treatment with some chemotherapeutic drugs, e.g., etoposide and bleomycin. The xrs-6 cells, which lack the $\mathrm{Ku} 80$, were shown to be sensitive to etoposide and bleomycin $[13,36]$. It was reported that plateau phase xrs- 6 cells were 500 -fold more sensitive to bleomycin treatment than were parental $\mathrm{CHO}-\mathrm{K} 1$ cells [36]. It was demonstrated that transfection of xrs- 6 cells with the $\mathrm{Ku} 80 \mathrm{cDNA}$ rescued X-ray sensitivity and chemosensitivity of the mutant cells $[13,33]$. In this study, we examined the localization and mobility of EGFP-Ku80 in living hamster xrs- 6 cells with or without DSBs, which were induced by treatment with etoposide or bleomycin.

\section{MATERIALS AND METHODS}

Cell lines and cultures: The Chinese hamster ovary mutant xrs- 6 was derived from the CHO-K1 cell line on the basis of their sensitivity to ionizing radiation [33]. The gene product defective in xrs- 6 has been identified as Ku80 [33]. The cell line of xrs- 6 cells stably expressing EGFP-Ku80 was described previously $[13,15]$. We have established cell line of CHO-K1 cells stably expressing GFP-H2AX. The cell line of human tumors, HeLa-S3, was described in previous studies [18]. Cells in the exponentially growing phase were exposed to various doses of etoposide and bleomycin. Both DNA DSB inducers were purchased from Wako Pure Chemical (Osaka, Japan).

Cell survival assays: Exponentially growing cells were plated at 500 cells/well in 96-well plates and subjected to cell survival assays. The following day, the cells were exposed to various concentrations of etoposide for $1 \mathrm{hr}$ at $37^{\circ} \mathrm{C}$ in media [13]. Etoposide was freshly diluted from a $68 \mathrm{mM}$ stock solution in DMSO. Cells exposed to etoposide were then washed twice with a medium, placed in a fresh medium and incubated for 2 days. The survival rates of the cells were monitored by MTS assay using CellTiter 96 Aqueous One Solution Cell Proliferation Assay (Promega, WI). All exposures were carried out in triplicate.

Immunoblotting: The extraction of total lysates was performed as previously described $[16,18,19]$. The supernatants were electrophoresed on 4-20\% SDS-polyacrylamide gels. The fractionated products were electrotransferred onto
Hybond-P membranes (GE Healthcare Bio-Sci. Corp., Piscataway, NJ). After blocking nonspecific binding sites with an ECL-blocking reagent (GE Healthcare Bio-Sci. Corp.), the membranes were incubated with a goat anti-Ku70 polyclonal antibody (C-19) (Santa Cruz Biotechnology, Santa Cruz, Ca), a goat anti-Ku80 polyclonal antibody (M-20) (Santa Cruz Biotechnology), a mouse anti- $\gamma-\mathrm{H} 2 \mathrm{AX}$ monoclonal antibody (Upstate Biotechnology Inc., Charlottesville, VA) or a mouse anti- $\beta$-actin monoclonal antibody (Sigma, St. Louis, MO). The corresponding proteins were visualized using an enhanced chemiluminescence (ECL) Western blotting detection system (GE Healthcare Bio-Sci. Corp.), in accordance with the manufacturer's instructions.

Immunofluorescence staining: Immunofluorescence staining was performed as previously described [17]. Briefly, cells were grown on culture glass slides (Falcon), washed with PBS, and fixed at room temperature. The fixed cells were first blocked for 30 minutes (min) using a blocking solution and then incubated for $30 \mathrm{~min}$ at room temperature with a goat anti-Ku80 polyclonal antibody (M-20) or a mouse anti- $\gamma-\mathrm{H} 2 \mathrm{AX}$ monoclonal antibody. After washing with PBS three times, antibody binding was detected by the application of Alexa fluor 568-conjugated secondary antibodies (Molecular Probes, OR). DNA was stained with the DAPI fluorescent dye. The distribution of fluorescent signals was monitored by FV300 confocal laser scanning microscope (Olympus, Tokyo, Japan) as previously described [15].

FRAP analysis using confocal laser scanning microscopy: FRAP analysis using confocal laser scanning microscopy was performed as previously described [34]. Briefly, images of live cells and time-series FRAP images were obtained by confocal laser scanning microscopy (ZeissLSM510 Meta, Carl Zeiss, Germany). Green fluorescence in the range from 505 to $550 \mathrm{~nm}$ was detected by the excitation at $488 \mathrm{~nm}$ with an argon laser. Photobleaching was carried out by 5 iterative scans of a selected region at the maximal laser power. Both of the images of the region which was photo-bleached and the region that was not photobleaced, were recorded before photobleaching, immediately after photobleaching, and subsequently every 0.8 seconds (sec), with 10\% laser power. The fluorescence intensity within the photobleached or unphotobleached region was measured in each frame and was calculated using software in LSM510. The reproducibility of all the data was confirmed by doing twice or more repeatedly.

\section{RESULTS}

Techniques using GFP are a powerful tool for analyzing protein mobility in living cells. To examine the localization and mobility of Ku80 in living cells, it is convenient to use cell lines stably expressing chimeric constructs of EGFP and the wild-type Ku80 protein. Previously, we established cell lines stably expressing EGFP-Ku80 (N-terminal tagging) using Ku80-deficient hamster xrs-6 cells [13, 15]. Firstly, to reconfirm whether EGFP-tagged $\mathrm{Ku} 80$ is expressed and 

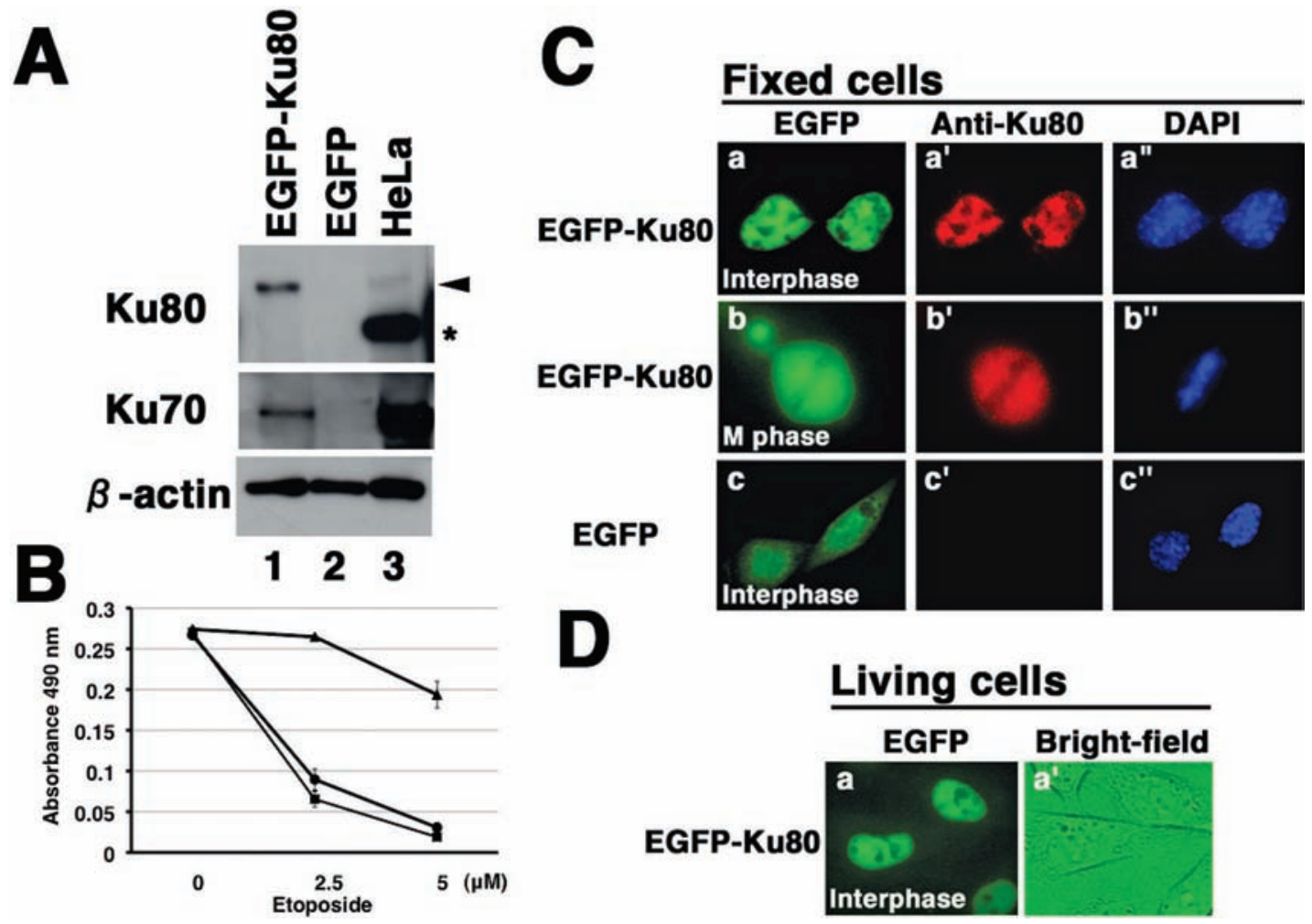

\section{Living cells}

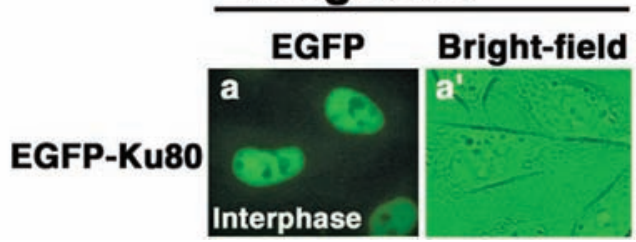

Fig. 1. Characterization of xrs-6 cells transformed with EGFP-tagged Ku80. A, Western blot analysis of xrs-6 cells constitutively expressing Ku80 tagged with EGFP or EGFP alone and human cells. Total cell lysates were analyzed by Western blotting using an anti-Ku80 antibody, an anti-Ku70 antibody or an anti- $\beta$-actin antibody. Lane 1, EGFP-Ku80-expressing xrs-6 cells (EGFP-Ku80). Lane 2, EGFP-expressing xrs-6 cells (EGFP). Lane 3, HeLa cells (HeLa). Arrowhead, Ku80 tagged with EGFP; Asterisk, human wild-type Ku80. B, Survival of xrs-6 cells transformed with EGFP-Ku80 following etoposide exposure. Untransfected xrs-6 cells (circle) or xrs-6 cells transformed with EGFP-Ku80 (triangle) or EGFP (square) were treated with $0,2.5$ and $5 \mu \mathrm{M}$ of etoposide. The number of viable cells was measured by MTS assay after 48 hr. Error bars denote standard deviations of triplicate measurements. C, Intracellular distribution of Ku80 in intact paraformaldehyde-fixed cells at interphase and metaphase. Xrs-6 cells constitutively expressing EGFP-Ku80 (a, a', a', b, b', b'") or EGFP (c, c', c'") were fixed and stained with DAPI (right) and anti-Ku80 antibody (middle). Interphase (a, a', a', c, c', c') and metaphase (M phase) (b, b', b") cells. Recorded using 40X (c-c'") and 60X objectives (a-a', b-b'). D, Intracellular distribution of Ku80 in living cells during interphase. Xrs-6 cells constitutively expressing EGFP-Ku80 (a, a') were analyzed by immunofluorescence microscopy. Bright-field images of the cells at interphase (a') are shown. The corresponding images for EGFP fluorescence are shown in the left panels (a). Recorded using a 60X objective.

functioned stably in the tranfectants, the expression levels of $\mathrm{Ku} 70$ and $\mathrm{Ku} 80$ in whole-cell extracts prepared from xrs-6 transfectants containing pEGFP or pEGFP-Ku80 were compared with those from human cells (HeLa). As shown in Fig. 1, EGFP-Ku80 in the transfectants was expressed at a lower level than endogenous Ku80 in HeLa cells, suggesting that EGFP-tagged Ku80 was not overexpressed abnormally in the transfectants. In addition, we detected that hamster $\mathrm{Ku} 70$ in extracts prepared from the EGFP-Ku80 transfectants was expressed, although the level was lower than that of endogenous $\mathrm{Ku} 70$ in extracts from HeLa cells (Fig. 1), confirming that exogenous Ku80 tagged with EGFP, as well as human Ku80 in HeLa cells, stabilizes endogenous $\mathrm{Ku} 70$ in these stable transfectants. Expectedly, we were not able to detect hamster $\mathrm{Ku} 70$ and $\mathrm{Ku} 80$ in the extracts prepared from the EGFP transfectants (Fig. 1A). In addition, we reconfirmed that EGFP-Ku80 in the transfectants can significantly complement the defects of etoposide sensitivity of xrs-6 cells (Fig. 1B). To compare the subcellular localization of EGFP-Ku80 during cell cycle in fixed and living cells, the localization of the fusion protein was examined under a fluorescence microscope. First, transfectants expressing either EGFP-Ku80 or EGFP alone were fixed and immunostained with an anti-Ku80 antibody, to confirm that the proteins visualized using EGFP were Ku80 tagged with EGFP in EGFP-Ku80 transfectants (Fig. 1C). We observed an agreement between Ku80 staining and the fluorescence of EGFP in the EGFP-Ku80 transfectants (Figs. 1Ca', b'). On the other hand, no Ku80 staining was observed in the EGFP transfectants (Fig. 1Cc'). In the both fixed and living cells, immunofluorescence microscopy showed that EGFP-Ku80 localized in the nuclei of inter- 
0.0

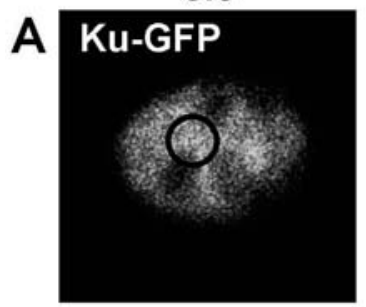

B

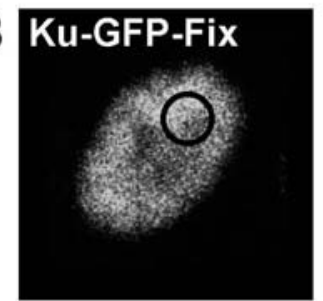

C

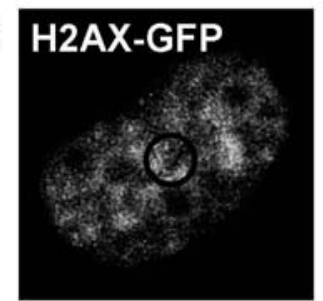

1.6
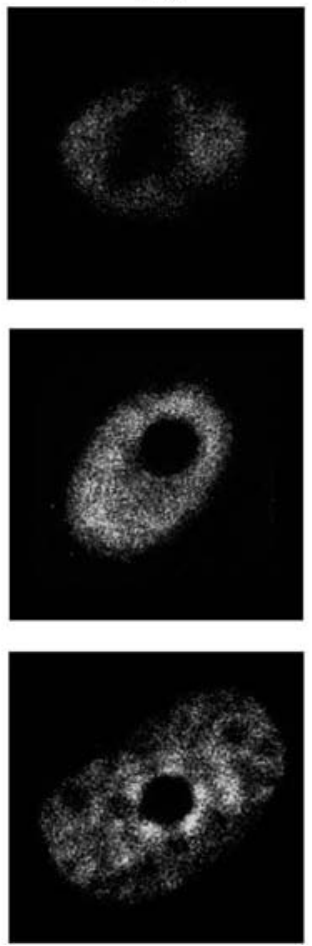

7.4
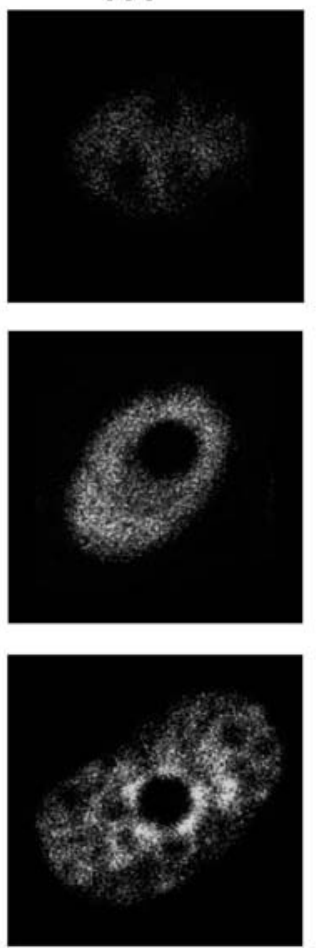

$20 \mathrm{sec}$
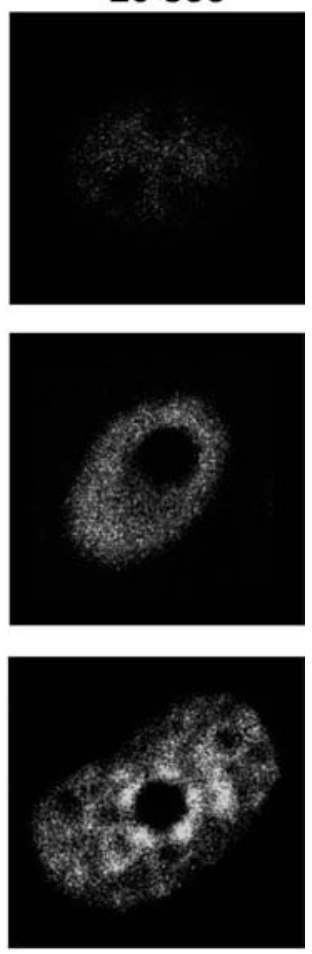

Fig. 2. FRAP analysis of diffusional mobility of EGFP-Ku80 in stable transfectants. The circles indicate the regions that were photobleached. The hamster cell in the middle panel (B) was fixed with methanol before FRAP analysis (Ku-GFP-Fix). Time points of recovery after photobleaching are indicated in sec. The recovery of EGFP-Ku80 within the photobleached region in living hamster cells was rapid (A), whereas the recovery of EGFP-Ku80 in fixed cells and that of GFP-H2AX in living hamster cells were not observed (B, C). (A) Ku-GFP, Living cells stably expressing EGFP-Ku80; (B) Ku-GFP-Fix, Fixed cells stably expressing EGFP-Ku80; (C) H2AX-GFP, Living cells stably expressing GFP-H2AX.

phase cells, whereas EGFP-Ku80 was distributed diffusely in the cytoplasm of mitotic cells (Figs. 1C and 1D) (data not shown). These data support the idea that the cell lines stably expressing EGFP-Ku80 are useful for the analysis of Ku80 behavior.

Next, to clarify the dynamic behavior of Ku80 in the nucleus of our established living hamster cells, we analyzed the mobility of EGFP-Ku80 in the nucleus of stable EGFP$\mathrm{Ku} 80$ transfectants using FRAP analysis. We found that fluorescence was recovered rapidly after bleaching in living hamster cells (Fig. 2A). In addition, we found that total fluorescence was decreased during photobleaching and sharply-defined bleaching area was not observed in living hamster cells (Fig. 2A), supporting the idea that EGFP$\mathrm{Ku} 80$ has high mobility. In addition, we analyzed the mobility of GFP-H2AX in the nucleus of stable GFP-H2AX transfectants using FRAP analysis. As shown in Fig. 2C, there was little or no fluorescence recovery $20 \mathrm{sec}$ after photobleaching in hamster cells stably expressing GFP-H2AX. Expectedly, no fluorescence was recovered in hamster cells stably expressing EGFP-Ku80, which were fixed before the photobleaching (Fig. 2B).

We determined the mobility of EGFP-Ku80 in our stably expressing cells. The change of fluorescence levels within both unbleached (Fig. 3A, b) and photobleached (Fig. 3A, a) regions were determined by scanning every $0.8 \mathrm{sec}$. As shown in Fig. 3B, the fluorescence within the photobleached region was recovered at the same intensity as that within the unphotobleached region in the nucleus of living hamster cells after photobleaching. On the other hand, the fluorescence intensity within the unphotobleached region decreased in the nucleus of living hamster cells after photobleaching, suggesting that EGFP-Ku80 has a high diffusional mobility. Expectedly, the recovery of fluorescence within the photobleached region and the change in fluorescence intensity within the unphotobleached region were not observed in hamster cells stably expressing GFP-H2AX (Fig. 3A, C).

Two drugs, namely, etoposide and bleomycin, are known to produce DNA DSBs with different efficiencies and by different mechanisms [35]. We first investigated the phosphorylation of $\mathrm{H} 2 \mathrm{AX}$ in the EGFP-Ku80 transfectants treated with the two drugs for $1 \mathrm{hr}$ by Western blot analysis using the anti- $\gamma-\mathrm{H} 2 \mathrm{AX}$ antibody. As shown in Fig. 4A, the level of H2AX phosphorylation on Ser 139 increased in response to etoposide or bleomycin in a dose-dependent 
A
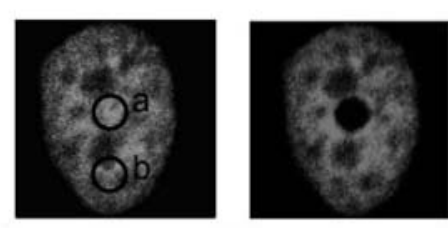

a: bleaching

b:unbleaching
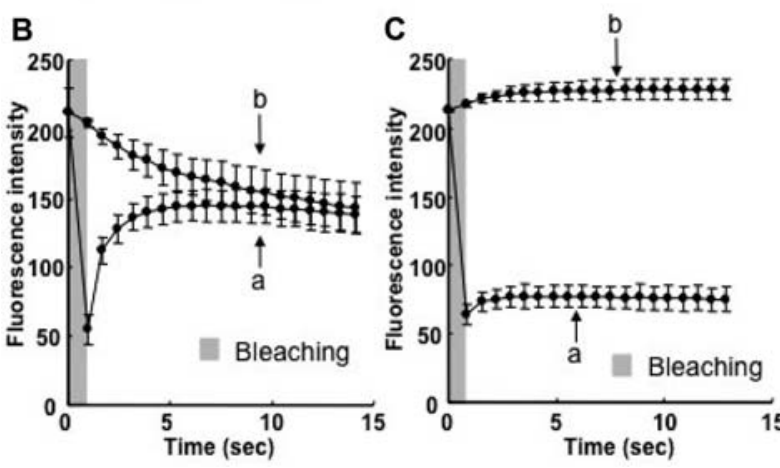

Fig. 3. Mobility of EGFP-Ku80 after photobleaching of nucleoplasmic area for $1 \mathrm{sec}$ (shadow). A, The circles indicate the regions that were photobleached (a) or unphotobleached (b) in cells stably expressing GFP-H2AX. Left panel, before photobleaching; Right panel, after photobleaching. B and C, FRAP analysis of EGFP-Ku80 or GFP-H2AX in stable transfectants. FRAP analysis of EGFP-Ku80 (B) and GFP-H2AX (C) in stable transfectants was performed using living cells. The fluorescence intensity within the photobleached (a) or unphotobleached (b) region was measured in each frame and was calculated using software in LSM510. Each data point is the average of 18 (B) or 5 (C) independent measurements. Error bars represent the SD.

manner.

It has not been clarify whether Ku80 accumulates and forms foci at DNA DSBs induced by etoposide or bleomycin, although Ku80 plays an essential role in the repair of DNA DSBs induced by these drugs in mammalian cells. In addition, it has not been determined whether the mobility of $\mathrm{Ku} 80$ is affected after treatment with these drugs. To clarify the localization and mobility of Ku80 under the conditions of induction of the phosphorylation of H2AX on Ser139 following the treatment with these DSB inducers, we first examined the localization of EGFP-Ku80 after treatment with etoposide or bleomycin for $1 \mathrm{hr}$ in the EGFP-Ku80 transfectants. As shown in Fig. 4B, a strong $\gamma-\mathrm{H} 2 \mathrm{AX}$ staining was observed in the nuclei of the transfectants upon treatment with the drugs. EGFP-Ku80 fluorescence, as well as $\gamma$-H2AX staining, was detected within the nuclei after treatment with etoposide or bleomycin. However, we were not able to detect the accumulation of EGFP-Ku80 at the $\gamma$ H2AX foci using conventional fluorescence microscopy and confocal laser microscopy (Figs. 4B and 4C).

To study the response of a major portion of Ku80 to damage in living animal cells, we analyzed the mobility of EGFP-Ku80 in the damaged nucleus of the transfectants using FRAP analysis. As shown in Fig. 5, the mobility was unaffected after treatment with etoposide or bleomycin, suggesting that a major portion of EGFP-Ku80 does not show altered mobility in the transfectants with a large number of DNA DSBs.

\section{DISCUSSION}

It is well known that $\mathrm{Ku}$ is a genome caretaker gene. In malignant cells, Ku80 plays a key role in the repair of DNA DSBs induced by treatment with chemotherapeutic drug [2]. It is well known that Ku80-dependent DNA repair pathway participates in the mechanisms of the chemoresistance. On the other hand, the control mechanisms underlying the localization of $\mathrm{Ku} 80$ play a key role in regulating the function of $\mathrm{Ku}$ in vivo $[12,31]$. Howevere, it has not been determined whether the localization and mobility of Ku80 is affected after treatment with these drugs. In this study, we examined the localization and mobility of Ku80 in living hamster cells with or without DSBs, which were induced by treatment with chemotherapeutic drugs. Our data showed that $\mathrm{Ku} 80$, in contrast to $\mathrm{H} 2 \mathrm{AX}$, is highly mobile in the absence of DSBs. In addition, we first noted that before and after the induction of DNA damage by treatment with etoposide or bleomycin, a major portion of Ku80 in stable transfectants is exchanged by the same kinetics in the nuclei of interphase cells.

Rodgers et al. has originally reported that transiently expressed Ku80-EGFP (C-terminal tagging) is highly mobile in human cancer cells [26]. On the other hand, immobility of H2AX in the absence of DNA damage was originally shown in human cells [30]. In this study, we confirmed the results concerning the high mobility of Ku80 in hamster xrs- 6 cells. In addition, we showed that $\mathrm{Ku} 80$, in contrast to $\mathrm{H} 2 \mathrm{AX}$, is highly mobile in the absence of DSBs in hamster xrs-6 cells. As described above, EGFP-Ku80 (Nterminal tagging) used in this study complemented not only $\mathrm{X}$-ray sensitivity but also the etoposide sensitivity of Ku80deficient hamster xrs-6 cells [13]. FRAP analysis using cells stably expressing EGFP-Ku80 suggests that functional EGFP-Ku80 (N-terminal tagging) is highly freely mobile in the absence of DSBs in endogenous Ku80-deficient hamster xrs- 6 cells. Recently, using these cells, we showed that EGFP-Ku80 accumulation at DSBs began immediately after irradiation using a microlaser [15]. Thus, Ku80 might be moving rapidly within the nuclei in order to find DSBs in hamster cells.

Bleomycin and etoposide induce DNA DSBs via different mechanisms and efficiencies [35]. It was reported that radiation-induced strand breaks are repaired with a half time of less than $5 \mathrm{~min}$, whereas etoposide-induced strand breaks are repaired with an hour or more [23]. EGFP-Ku80 used in this study complemented not only X-ray sensitivity but also the etoposide sensitivity of Ku80-deficient cells [13], although the rates of rejoining of DNA DSBs differ between etoposide and radiation [23]. It is well known that phspohorylation of $\mathrm{H} 2 \mathrm{AX}$ is a sensitive indicator of DNA DSBs induced by drugs, e.g., bleomycin and etoposide, as well as 

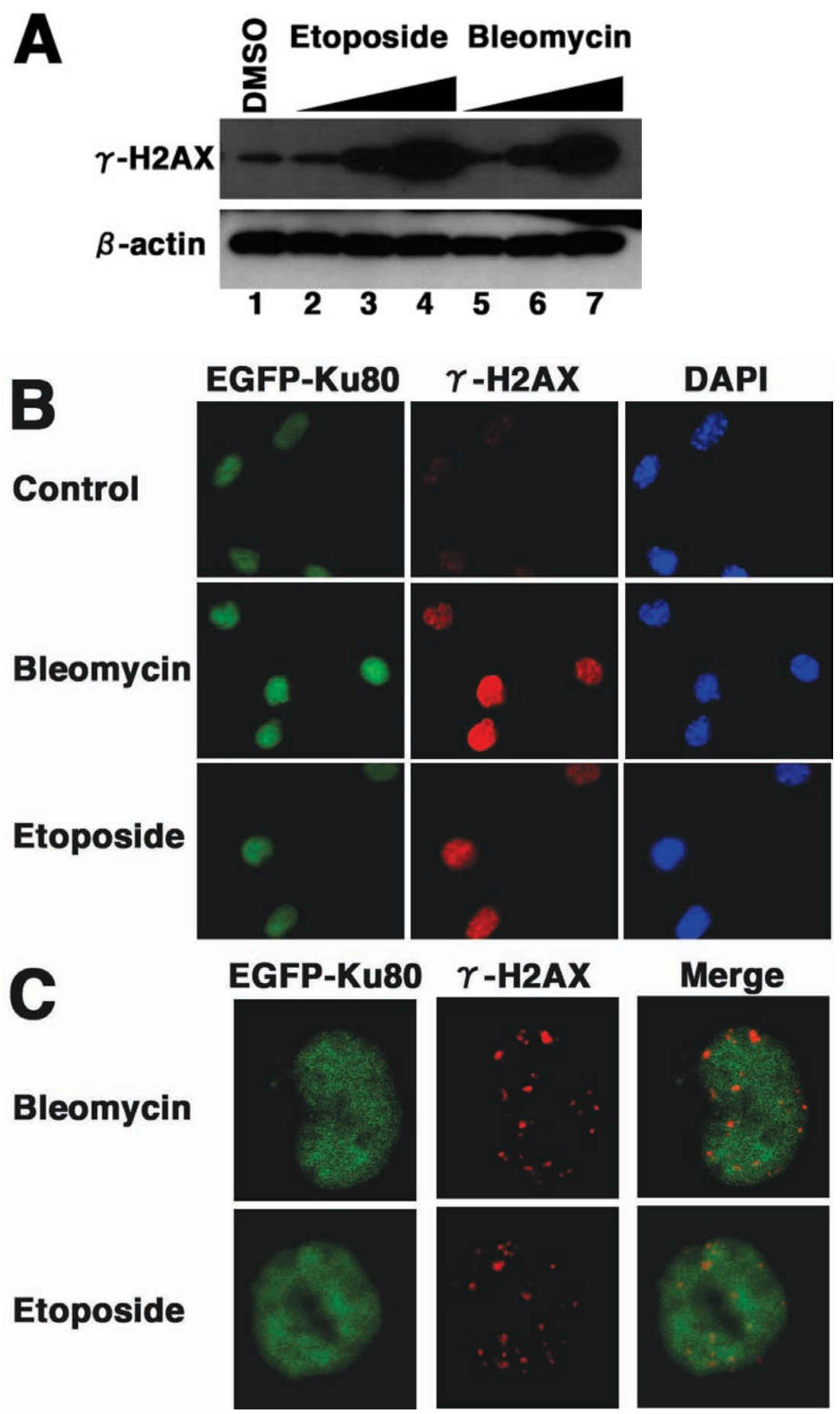

Fig. 4. Accumulation of $\mathrm{H} 2 \mathrm{AX}$ phosphorylation in xrs-6 cells constitutively expressing EGFP-Ku 80 as induced by bleomycin or etoposidein treatment. A, Cells were treated with etoposide $(0.25,2.5$ and $25 \mu \mathrm{M})$ or bleomycin $(0.1,1,10 \mu \mathrm{g} / \mathrm{m} l)$ for $1 \mathrm{hr}$. Total cell lysates were analyzed by Western blotting using an anti- $\gamma-\mathrm{H} 2 \mathrm{AX}$ antibody or an anti- $\beta$-actin antibody. Lane 1, DMSO (as a control); Lane 2, etoposide $(0.25 \mu \mathrm{M})$; Lane 3, etoposide (2.5 $\mu \mathrm{M})$; Lane 4, etoposide (25 $\mu \mathrm{M})$; Lane 5, bleomycin $(0.1 \mu \mathrm{g} / \mathrm{m} l)$; Lane 6, bleomycin $(1 \mu \mathrm{g} / \mathrm{m} l)$; Lane 7, bleomycin $(10 \mu \mathrm{g} / \mathrm{m} l)$. B and C, Cells were treated with etoposide $(25 \mu \mathrm{M})$, bleomycin $(10 \mu \mathrm{g} / \mathrm{m} l)$ or DMSO (control) for $1 \mathrm{hr}$. Then, the cells were fixed and stained with an anti$\gamma$-H2AX antibody and DAPI. The subcellular localization of the EGFP-tagged proteins (green), $\gamma$-H2AX (red) and DAPI (blue) was examined in the same cells by conventional fluorescence microscopy (B) or confocal laser microscopy (C). 

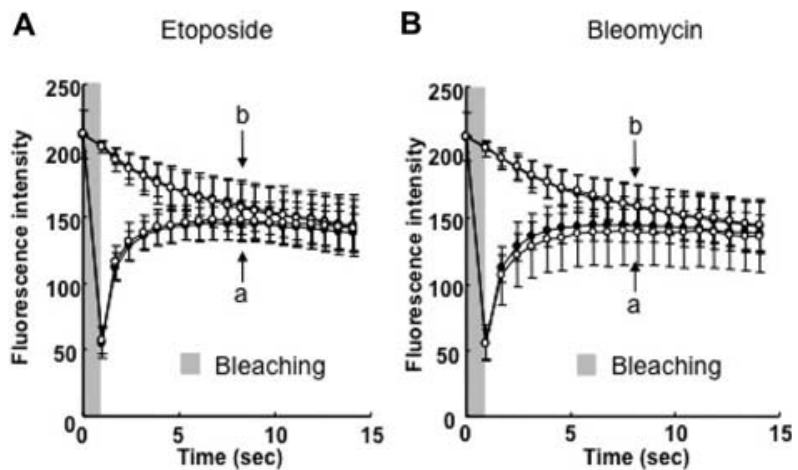

Fig. 5. Mobility of EGFP-Ku80 after photobleaching of a nucleoplasmic area for $1 \mathrm{sec}$ (shadow) with or without treatment with DSB inducer. Treated cells were exposed to etoposide $(25 \mu \mathrm{M})$ (A) or bleomycin $(10 \mu \mathrm{g} / \mathrm{m} l)$ (B) for $1 \mathrm{hr}$. The fluorescence intensity within the photobleached (a) or unphotobleached (b) region was measured at about $0.8 \mathrm{sec}$ intervals after photobleaching ( $\bigcirc$, untreated; $\bigcirc$, treated). Each data point is the average of 18 (A) or 13 (B) independent measurements. Error bars represent the SD.

by radiation [35]. In this study, as $\mathrm{H} 2 \mathrm{AX}$ was phosphorylated $1 \mathrm{hr}$ after treatment with the reagents, a large number of DSBs were certainly formed at the concentrations we used. Thus, our data suggest that the generation of a large number of DSBs by treatment with 2 chemotheraputic drugs, i.e., etoposide or bleomycin do not affect the localization and mobility of a major portion of EGFP-Ku80. We speculate that the mobility of a major portion of Ku80 is not affected by DNA DSBs in order to find other DSBs.

Previously, we reported that the accumulation of EGFPKu 80 at microlaser-induced DNA DSBs $(\gamma-\mathrm{H} 2 \mathrm{AX}$ foci) has been detected easily using the detection conditions used in this study [15]. However, under the same detection conditions, whereas $\gamma$-H2AX foci has been detected easily, the accumulation of EGFP-Ku 80 has not been detected at DNA DSBs $(\gamma-\mathrm{H} 2 \mathrm{AX}$ foci) and EGFP-Ku80 did not change its pattern of localization cytologyically following treatment with etoposide and bleomycin. On the other hand, the localization of NHEJ factors, e.g., Ku70 and Ku80, at ionizing radiation (IR)-induced DNA DSBs ( $\gamma$-H2AX foci) has not been cytologically detected, but the localization of HR- and HR-related factors at IR-induced DNA DSBs $(\gamma-\mathrm{H} 2 \mathrm{AX}$ foci), e.g., $\operatorname{Rad} 51, \operatorname{Rad} 52, \mathrm{Nbs} 1$, and 53BP1, has been detected easily $[10,20,22]$. Thus, we conclude that the accumulation of Ku80 to a level that they become visible as a nuclear focus at etoposide- or bleomycin-induced damage sites as well as at IR-induced damage sites, is not required in the repair of a single DSB through the NHEJ pathway. On the other hand, the differences of results between our previous microlaser study [15] and this drug study might be depend on the difference by both the complexity of DSBs and the density of DSBs, although further studies are required to clarify this.

In conclusion, we have shown the localization and mobility of EGFP-Ku80 in living animal cells with or without DSBs, which were induced by treatment with chemotherapeutic drugs. Further studies to elucidate the molecular mechanism underlying the mobility and localization of $\mathrm{Ku}$ will lead to a better understanding of not only the regulation mechanism of this protein, but also of the development of new gene therapy and chemotherapy to many types of animal malignancies.

ACKNOWLEDGMENTS. We thank Dr. P. Jeggo for providing xrs-6 cells. We also thank Dr. N.V. Tomilin for providing GFP-H2AX plasmid vector. We also thank Mr. Y. Y. for his technical assistance with the immunoblotting. This work was supported in part by grants from the Ministry of Education, Science, Sports, and Culture, Japan. Part of this study was financially supported by the Budget for Nuclear Research of the Ministry of Education, Culture, Sports, Sciences and Technology, based on the screening and counseling by the Atomic Energy Commission.

\section{REFERENCES}

1. Banath, J. P. and Olive, P. L. 2003. Expression of phosphorylated histone $\mathrm{H} 2 \mathrm{AX}$ as a surrogate of cell killing by drugs that create DNA double-strand breaks. Cancer Res. 63: 4347-4350.

2. Belenkov, A. I., Paiemen, J. P., Panasci, L. C., Monia, B. P. and Chow, T. Y. 2002. An antisense oligonucleotide targeted to human Ku86 messenger RNA sensitizes M059K malignant glioma cells to ionizing radiation, bleomycin, and etoposide but not DNA cross-linking agents. Cancer Res. 62: 5888-5896.

3. Celeste, A., Petersen, S., Romanienko, P. J., FernandezCapetillo, O., Chen, H. T., Sedelnikova, O. A., Reina-SanMartin, B., Coppola, V., Meffre, E., Difilippantonio, M. J., Redon, C., Pilch, D. R., Olaru, A., Eckhaus, M., CameriniOtero, R. D., Tessarollo, L., Livak, F., Manova, K., Bonner, W. M., Nussenzweig, M. C. and Nussenzweig, A. 2002. Genomic instability in mice lacking histone H2AX. Science 296: 922927.

4. Celeste, A., Fernandez-Capetillo, O., Kruhlak, M. J., Pilch, D. R., Staudt, D. W., Lee, A., Bonner, R. F., Bonner, W. M. and Nussenzweig, A. 2003. Histone H2AX phosphorylation is dispensable for the initial recognition of DNA breaks. Nat. Cell Biol. 5: 675-679.

5. Collis, S. J., DeWeese, T. L., Jeggo, P. A. and Parker, A. R. 2005. The life and death of DNA-PK. Oncogene 24: 949-961.

6. Ding, Q., Bramble, L., Yuzbasiyan-Gurkan, V., Bell., T. and Meek, K. 2002. DNA-PKcs mutations in dogs and horses: allele frequency and association with neoplasia. Gene $\mathbf{2 8 3}$ : 263-269.

7. Ferguson, D., Sekiguchi, J., Chang, S., Frank, K., Gao, Y., DePinho, R. A. and Alt, F. W. 2000. The nonhomologous endjoining pathway of DNA repair is required for genomic stability and the suppression of translocations. Proc. Natl. Acad. Sci. U.S.A. 97: 6630-6633.

8. Gullo, C., Au, M., Feng, G. and Teoh, G. 2006. The biology of $\mathrm{Ku}$ and its potential oncogenic role in cancer. Biochim. Biophys. Acta 1765: 223-234.

9. Hopfner, K. P., Putnam, C. D. and Tainer, J. A. 2002. DNA double-strand break repair from head to tail. Curr. Opin. Struct. Biol. 12: 115-122.

10. Jakob, B., Scholz, M. and Taucher-Scholz, G. 2002. Character- 
ization of CDKN1A (p21) binding to sites of heavy-ioninduced damage: colocalization with proteins involved in DNA repair. Int. J. Radiat. Biol. 78: 75-88.

11. Khanna, K. K. and Jackson, S. P. 2001. DNA double-strand breaks: signaling, repair and the cancer connection. Nat. Genet. 27: $247-254$.

12. Koike, M. 2002. Dimerization, translocation and localization of Ku70 and Ku80 proteins. J. Radiat. Res. 43: 223-236.

13. Koike, M. and Koike, A. 2004. Establishment and characterization of stable cell lines expressing human Ku80 tagged with enhanced green fluorescent protein. J. Radiat. Res. 45: 119125.

14. Koike, M. and Koike, A. 2005. The Ku70-binding site of Ku80 is required for the stabilization of $\mathrm{Ku} 70$ in the cytoplasm, for the nuclear translocation of $\mathrm{Ku} 80$, and for Ku80-dependent DNA repair. Exp. Cell Res. 305: 266-276.

15. Koike, M. and Koike, A. 2008. Accumulation of Ku80 proteins at DNA double-strand breaks in living cells. Exp. Cell Res. 314: 1061-1070.

16. Koike, M., Ikuta, T., Miyasaka, T. and Shiomi, T. 1999. Ku80 can translocate to the nucleus independent of the translocation of Ku70 using its own nuclear localization signal. Oncogene 18: 7495-7505.

17. Koike, M., Ikuta, T., Miyasaka, T. and Shiomi, T. 1999. The nuclear localization signal of the human $\mathrm{Ku} 70$ is a variant bipartite type recognized by the two components of nuclear pore-targeting complex. Exp. Cell Res. 250: 401-413.

18. Koike, M., Miyasaka, T., Mimori, T. and Shiomi, T. 1998. Subcellular localization and protein-protein interaction regions of Ku proteins. Biochem. Biophys. Res. Commun. 252: 679685.

19. Koike, M., Awaji, T., Kataoka, M., Tsujimoto, G., Kartasova, T., Koike, A. and Shiomi, T. 1999. Differential subcellular localization of DNA-dependent protein kinase components $\mathrm{Ku}$ and DNA-PKcs during mitosis. J. Cell Sci. 112: 4031-4039.

20. Kim, J. S., Krasieva, T. B., Kurumizaka, H., Chen, D. J., Taylor, A. M. and Yokomori, K. J. 2005. Independent and sequential recruitment of NHEJ and HR factors to DNA damage sites in mammalian cells. J. Cell Biol. 170: 341-347.

21. Lees-Miller, S. P. 1996. The DNA-dependent protein kinase, DNA-PK: 10 years and no ends in sight. Biochem. Cell Biol. 74: $503-512$.

22. Lisby, M., Barlow, J. H., Burgess, R. C. and Rothstein, R. 2004. Choreography of the DNA damage response: spatiotemporal relationships among checkpoint and repair proteins. Cell 118: 699-713.

23. Olive, P. L. and Johnston, P. J. 1997. DNA damage from oxidants: influence of lesion complexity and chromatin organization. Oncol. Res. 9: 287-294.
24. Paull, T. T., Rogakou, E. P., Yamazaki, V., Kirchgessner, C. U., Gellert, M. and Bonner, W. M. 2000. A critical role for histone $\mathrm{H} 2 \mathrm{AX}$ in recruitment of repair factors to nuclear foci after DNA damage. Curr. Biol. 10: 886-895.

25. Perryman, L. E. 2004. Molecular pathology of severe combined immunodeficiency in mice, horses, and dogs. Vet. Pathol. 41: 95-100.

26. Rodgers, W., Jordan, S. J. and Capra, J. D. 2002. Transient association of $\mathrm{Ku}$ with nuclear substrates characterized using fluorescence photobleaching. J. Immunol. 168: 2348-2355.

27. Rogakou, E. P., Pilch, D. R., Orr, A. H., Ivanova, V. S. and Bonner, W. M. 1998. DNA double-stranded breaks induce histone H2AX phosphorylation on serine 139. J. Biol. Chem. 273: 5858-5868.

28. Rogakou, E. P., Boon, C., Redon, C. and Bonner, W. M. 1999. Megabase chromatin domains involved in DNA double-strand breaks in vivo. J. Cell Biol. 146: 905-916.

29. Shiloh, Y. 2003. ATM and related protein kinases: safeguarding genome integrity. Nat. Rev. Cancer 3: 155-168.

30. Siino, J. S., Nazarov, I. B., Svetlova, M. P., Solovjeva, L. V., Adamson, R. H., Zalenskaya, I. A., Yau, P. M., Bradbury, E. M. and Tomilin, N. V. 2002. Photobleaching of GFP-labeled H2AX in chromatin: H2AX has low diffusional mobility in the nucleus. Biochem. Biophys. Res. Commun. 297: 1318-1323.

31. Song, Y., Lim, J. W., Kim, H., Morio, T. and Kim, K. H. 2003. Oxidative stress induces nuclear loss of DNA repair proteins $\mathrm{Ku} 70$ and $\mathrm{Ku} 80$ and apoptosis in pancreatic acinar AR42J cells. J. Biol. Chem. 278: 36676-36687.

32. Stiff, T., O'Driscoll, M., Rief, N., Iwabuchi, K., Lobrich, M. and Jeggo, P. A. 2004. ATM and DNA-PK function redundantly to phosphorylate $\mathrm{H} 2 \mathrm{AX}$ after exposure to ionizing radiation. Cancer Res. 64: 2390-2396.

33. Taccioli, G. E., Gottlieb, T. M., Blunt, T., Priestley, A., Demengeot, J., Mizuta, R., Lehmann, A. R., Alt, F. W., Jackson, S. P. and Jeggo, P. A. 1994. Ku80: product of the XRCC5 gene and its role in DNA repair and V(D)J recombination. Science 265: 1442-1445.

34. Toyooka, T., Ibuki, Y., Koike, M., Ohashi, N., Takahashi, S. and Goto, R. 2004. Coexposure to benzo[a]pyrene plus UVA induced DNA double strand breaks: visualization of $\mathrm{Ku}$ assembly in the nucleus having DNA lesions. Biochem. Biophys. Res. Commun. 322: 631-636.

35. Watters, G. P., Smart, D. J., Harvey, J. S. and Austin, C. A. 2009. H2AX phosphorylation as a genotoxicity endpoint. Mutat. Res. 679: 50-58.

36. Zhou, T. and Povirk, L. F. 2005. Extreme cytotoxicity and susceptibility to hprt mutagenesis in Ku-deficient xrs- 6 cells treated with bleomycin in plateau phase. Mutagenesis 20: 39 44. 